\title{
Risikostratifizierung ausgewählter schwerer Organbeteiligungen bei Systemischer Sklerose und bei Lupus-Nephritis
}

\section{Risk Stratification of Selected Severe Organ Manifestations in Systemic Sclerosis and in Lupus Nephritis}

Autoren

Peter Oelzner ${ }^{1}$, Gunter Wolf²

Institute

1 Rheumatologie/Osteologie, Klinik für Innere Medizin III, Universitätsklinikum Jena, Jena, Deutschland

2 Nephrologie, Klinik für Innere Medizin III, Universitätsklinikum Jena , Jena, Deutschland

Schlüsselwörter

Risikostratifizierung, Systemische Sklerose, Lupusnephritis

Key words

risk stratification, systemic sclerosis, lupus nephritis

Bibliografie

Akt Rheumatol 2021; 46: 168-178

DOI $10.1055 / a-1403-5137$

ISSN 0034-3536

(C) 2021. Thieme. All rights reserved.

Georg Thieme Verlag KG, Rüdigerstraße 14,

70469 Stuttgart, Germany

Korrespondenzadresse

Peter Oelzner

Klinik für Innere Medizin III

Universitätsklinikum Jena

Erlanger Allee 101

07740 Jena

Deutschland

Tel.: + 49/3641/9324326, Fax: + 49/3641/9326847

Peter.Oelzner@med.uni-jena.de

\section{ZUSAMMENFASSUNG}

Interstitielle Lungenerkrankung (ILD), pulmonal-arterielle Hypertonie (PAH), kardiale Beteiligung und renale Krise sind schwere Prognose-bestimmende Manifestationen der Systemischen Sklerose (SSc). Digitale Ulcerationen führen zu erheblicher Beeinträchtigung von Erwerbsfähigkeit und Lebensqualität. Im Rahmen der Betreuung von SSc-Patienten ist daher eine Risikostratifizierung in Hinblick auf Entwicklung und Progression dieser schweren Manifestationen von wesentlicher Bedeutung. Risikofaktoren für die Entwicklung einer SSc-ILD sind diffuse cutane Sklerodermie (dcSSc), männliches Ge- schlecht und der Nachweis von Topoisomerase-I-Antikörpern. Ausmaß und Verlauf der SSc-ILD sind variabel. Klinik, Ausgangsbefund und Dynamik der Lungenfunktion und hochauflösendes CT (HR-CT) des Thorax werden genutzt, um frühzeitig Patienten zu identifizieren, welche einer Immunsuppression bedürfen und von jenen zu unterscheiden, bei denen zunächst engmaschige Verlaufskontrollen vertretbar sind. Zu den Risikofaktoren einer SSc-PAH zählen langer Krankheitsverlauf, hohes Lebensalter bei Beginn der SSc, schwere Raynaud-Symptomatik, schwere digitale Ischämien sowie Teleangiektasien, der Nachweis von Centromer-Antikörpern sowie Antikörpern gegen Endothelin-A-Rezeptor und Angiotensin-1-Rezeptor und Hyperurikämie. Bei etablierter PAH erfolgt die Risikostratifizierung auf Basis der kalkulierten 1-Jahres-Mortaliät. Zur Kalkulation der 1-Jahresmortalität werden anamnestische und klinische Parameter wie Symptomprogression, Auftreten von Synkopen, Vorhandensein klinischer Zeichen einer Rechtsherzinsuffizienz, funktionelle WHO-Klasse, funktionsdiagnostische, laborchemische, echokardiografische sowie hämodynamische Parameter herangezogen. Nach den aktuellen Empfehlungen zur Therapie der PAH ist eine primäre Kombinationstherapie zumindest ab WHO-Funktionsklasse III und einem intermediären Risiko (1-Jahres-Mortalität $\geq 5 \%$ indiziert. Wesentliche Risikofaktoren einer kardialen Beteiligung bei SSc sind höheres Lebensalter, dcSSc und der Nachweis von Topoisomerase I-Antikörpern. Zu den Risikofaktoren der renalen Krise bei SSc zählen dcSSc, männliches Geschlecht, der Nachweis von RNA-Polymerase IIIAntikörpern, vorbestehende Proteinurie, aber auch eine Vortherapie mit Glukokortikoiden, ACE-Hemmern und Cyclosporin. Digitale Ulcera (DU) treten bei ca. 50\% der Patienten auf. Risikofaktoren von DU sind dcSSc, Nachweis von Topoisomerase I-Antikörpern, früher Beginn der Raynaud-Symptomatik, hoher Rodnan Skin Score und männliches Geschlecht. Entscheidendes Therapieziel bei der Lupus-Nephritis (LN) ist der Erhalt einer normalen Nierenfunktion und die Vermeidung einer terminalen Niereninsuffizienz, welche mit einer erhöhten Letalität assoziiert ist. Zu den Risikofaktoren eines ungünstigen Langzeitverlaufs der LN zählen v. a. eine initiale irreversible Nierenfunktionseinschränkung im Zusammenhang mit irreversiblen chronischen Läsionen in der Nierenbiopsie, unzureichend kontrollierte arterielle Hypertonie, ausgeprägte initiale Proteinurie und männliches 
Geschlecht. Eine effektive Reduktion der Proteinurie auf $<0,5-$ $0,8 \mathrm{~g} / \mathrm{d}$ innerhalb von 12 Monaten nach Beginn der Remissionsinduktion signalisiert dagegen eine günstige Prognose. Frühestmögliche Diagnose der LN und umgehende auf dem Befund der Nierenbiopsie basierende Therapie sowie Reinduktion bei ausbleibender Remission sind entscheidend für eine Minimierung der Risikos.

\section{ABSTRACT}

Interstitial lung disease (ILD), pulmonary arterial hypertension (PAH), cardiac involvement and renal crisis are critical manifestations of systemic sclerosis (SSc) that determine prognosis. Digital ulcers result in substantial impairment of ability to work and quality of life. In the course of management of SSc patients, risk stratification with respect to development and progression of severe manifestations is of critical importance. Risk factors for development of SSc-ILD are diffuse cutaneous scleroderma (dcSSc), male gender and the evidence of topoisomerase Iantibodies. Extent and course of SSc-ILD are variable. Clinical parameters, baseline findings and dynamics of pulmonary function tests and high-resolution CT (HR-CT) are used to identify patients requiring early immunosuppression and distinguish them from patients for whom short-term controls are sufficient. Long disease course, older age at the beginning of SSc, severe Raynaud syndrome and digital ischemia, teleangiectasia, the evidence of centromer antibodies and of antibodies against endothelin A receptor and angiotensin- 1 receptor and hyperuricemia are among the factors associated with an increased risk for SSc-PAH. In established PAH, risk stratification is based on calculated 1-year mortality. For calculation of 1-year mortality, historical and clinical parameters such as progression of symptoms, occurrence of syncopes, clinical signs of right heart failure, functional WHO class, clinical chemistry, echocardiographic and haemodynamic findings and results of function tests are used. According to the present recommendations for treatment of $\mathrm{PAH}$, primary combination therapy is indicated at least from WHO functional class III and intermediate risk (1-year mortality $>5 \%$ ). Important risk factors of cardiac involvement in SSc are older age, dcSSc and evidence of topoisomerase I-antibodies. Risk factors for SSc renal crisis include dcSSc, male gender, the evidence of anti-RNA polymerase III antibodies, pre-existing proteinuria but also pre-existing therapy with glucocorticoids, ACE inhibitors and cyclosporine. Digital ulcers (DU) occurs in about $50 \%$ of SSc patients. Risk factors of DU are dcSSc, evidence of topoisomerase I-antibodies, early onset Raynaud phenomenon, high Rodnan skin score and male gender. Preservation of renal function and prevention of end-stage renal disease that is associated with increased mortality are important aims of the therapy of lupus nephritis (LN). Risk factors for poor long-term outcome of LN are initial irreversible reduction in renal function in association with irreversible chronic lesions in renal biopsy, uncontrolled arterial hypertension, high initial proteinuria and male gender. On the other hand, effective reduction of proteinuria to $<0.5-0.8 \mathrm{~g}$ daily within 12 months after start of remission induction indicate a good prognosis. Earliest possible diagnosis of LN and urgent therapy based on the result of renal biopsy and re-induction in the case of no remission are critical for risk minimisation.

\section{Einleitung}

Systemische Sklerose (SSc) und Systemischer Lupus erythematodes (SLE) gehen häufig mit schwerwiegenden Organmanifestationen einher. Für eine Verbesserung der Prognose im Hinblick auf Mortalität und Erhalt der Organfunktion sowie zum Erhalt von Erwerbsfähigkeit und Lebensqualität sind die frühzeitige Erkennung dieser schwerwiegenden Manifestationen und eine umgehende adäquate Therapie von entscheidender Bedeutung. Dies erfordert engmaschige Kontrolluntersuchungen, welche insbesondere auf die frühzeitige Erkennung schwerer Manifestationen und aggressiver Verläufe zu einem Zeitpunkt zielen, zu dem diese einer erfolgversprechenden Therapie noch zugänglich sind und irreversible Schäden noch nicht aufgetreten sind bzw. sich in Grenzen halten. In diesem Zusammenhang dient eine individuelle Risikostratifizierung dem Ziel, frühzeitig Patienten mit besonderen Risiken für schwere Manifestationen zu erkennen und Kontrolluntersuchungen entsprechend zu fokussieren und ggf. zu verdichten. Ferner ist zu bedenken, daß die Verläufe von Organmanifestationen bei SSc und SLE sehr variabel sind. Progrediente Verläufe mit rascher Entwicklung von Organinsuffizienzen sind von jenen mit geringerer Dynamik zu unterscheiden. Diese Differenzierung dient der zügigen Einleitung einer aggressiven Therapie einerseits und der Vermeidung von Übertherapie mit potenziellen Nebenwirkungen andererseits. Prognose-bestimmende schwere Organmanifestationen bei SSc sind interstitielle Lungenerkrankung, pulmonal-arterielle Hypertonie, kardiale Beteiligung und renale Krise. Digitale Ulcerationen bedingen eine erhebliche Einschränkung von Lebensqualität und Erwerbsfähigkeit und sind häufig mit anderen schweren SScManifestationen assoziiert. Unter Berücksichtigung von Häufigkeit und prognostischer Bedeutung ist die Lupusnephritis die wichtigste Organmanifestation des SLE. Sowohl zu o.g. SSc-Manifestationen als auch zur Lupusnephritis sind umfangreiche Daten zur Risikostratifizierung erhoben worden, welche gut in der Praxis angewendet werden können. Aufgrund der klinischen Relevanz der o.g. SSc-Manifestationen und der Lupusnephritis sowie der guten Datenlage zur Risikostratifizierung erfolgt in der vorliegenden Übersicht eine Fokussierung auf diese Krankheitsbilder.

\section{Teil 1: Risikostratifizierung schwerer Organbeteiligungen bei Systemischer Sklerose}

Interstitielle Lungenerkrankung (ILD) und pulmonal-arterielle Hypertonie (PAH) sind die beiden entscheidenden Prognose-bestimmenden Manifestationen der Systemischen Sklerose (SSc) [1, 2]. Daher sind sowohl die Kenntnis der Risikofaktoren für die Entwicklung einer ILD sowie einer PAH als auch die Risikostratifizierung bei bereits etablierter ILD oder PAH von entscheidender Bedeutung für die adäquate Dichte von Kontrolluntersuchungen und das therapeutische Vorgehen. 


\section{Interstitielle Lungenerkrankung bei systemischer Sklerose}

Die interstitielle Lungenerkrankung (ILD) ist die häufigste lebensbedrohliche Organmanifestation der SSc. Basierend auf Untersuchungen mittels High-resolution CT (HRCT) liegt die Prävalenz der ILD bei SSc zwischen 47 und 84\% [1]. Hinweise auf eine ILD fanden sich in kanadischen Registerdaten in $65 \%$ im HRCT, in $26 \%$ bei der klinischen Untersuchung und in $22 \%$ in der konventionellen Röntgenaufnahme des Thorax [3]. Autoptisch läßt sich eine ILD sogar bei $74 \%$ der SSc-Patienten nachweisen [1]. In der EUSTAR-Datenbank zeigten 53,4\% der Patienten mit diffuser cutaner SSc (dcSSc) und 34,7\% mit limitierter cutaner SSc (ICSSC) eine ILD [4]. Im Zeitraum zwischen 1972 und 2002 hat die Bedeutung der renalen Krise als Todesursache von SSc-Patienten von 42 auf $6 \%$ abgenommen, während die ILD als Todesursache bei SSc von 6 auf 33 \% zugenommen hat [5]. Eine respiratorische Insuffizienz basierend auf der ILD war für 43 \% der Todesfälle bei SSc ursächlich [6]. Als ursächlich für die abnehmende Bedeutung der renalen Krise als Todesursache bei SSc sind die konsequente Therapie mit ACE-Hemmern sowie die Vermeidung höher dosierter Glukokortikoide bei SSc-Patienten von wesentlicher Bedeutung [5].

Als Risikofaktoren für die Entwicklung einer ILD konnten das Vorliegen einer dcSSc, männliches Geschlecht, Afro-karibische Ethnizität und der Nachweis von Topoisomerase I-Antikörpern (Scl-70AK) sowie das Fehlen von Centromer-Antikörpern identifiziert werden $[1,7,8]$. In der Lungenfunktionsdiagnostik sind eine Reduktion der forcierten Vitalkapazität (FVC) und der Diffusionskapazität der Lunge für Kohlenmonoxid (DLCO) wegweisend für eine ILD [1].

Der Verlauf bzw. die Progredienz der ILD bei SSc ist hochvariabel. Neben der Kenntnis der Risikofaktoren einer ILD ist daher die frühzeitige Erkennung einer Progredienz derselben bzw. die Kenntnis von Risikofaktoren der Progredienz entscheidend für die Therapiestrategie. Risikofaktoren für eine Progredienz der ILD sind männliches Geschlecht, dcSSc, Nachweis von Topoisomerase-I-AK, nukleoläres ANA-Muster, der Nachweis von anti-Th/To- und U3RNPAK, aber auch paraklinische Entzündungszeichen wie Erhöhung von CrP und IL-6, eine initiale Verminderung der FVC auf $<70 \%$ und/ oder der DLCO auf $<55 \%$ und eine Beteiligung von $>20 \%$ des Lungenvolumens $[1,8,9]$. Als weitere Biomarker einer Progredienz werden derzeit verschiedene Chemokin-Liganden und-Rezeptoren sowie Zytokine untersucht, wobei diese in der Regel in der Routinediagnostik noch nicht verfügbar sind [1, 8, 9]. Ausgewählte Risikofaktoren einer Progredienz der SSc-ILD sind in > Abb. 1 dargestellt. Obwohl keine einheitliche Definition für eine progrediente SSc-ILD existiert, hat sich in den letzten Jahren in erster Linie eine auf der Entwicklung der Lungenfunktion basierende Einteilung in eine progrediente bzw. stabile Erkrankung etabliert. Als Zeichen einer Progression gelten eine Abnahme der FVC um $\geq 10 \%$ gegenüber dem Ausgangswert oder eine Abnahme der FVC von 5-9\% in Verbindung mit einer Abnahme der DLCO von $\geq 15 \%$ innerhalb eines Jahres $[1,8]$. Eine derartige Dynamik der genannten Lungenfunktionsparameter war der beste 1-Jahresprädiktor für spätere Mortalität [10].

Ein relativ einfaches Staging-System zur Einschätzung der Prognose der ILD bei SSc wurde 2008 von Goh et al. vorgestellt [11]. Basierend auf der Mortalität und der Entwicklung der Lungenfunktion bei 330 SSc-Patienten wurde die prognostische Bedeutung der initialen HRCT- und Lungenfunktionsbefunde für den weiteren Ver-

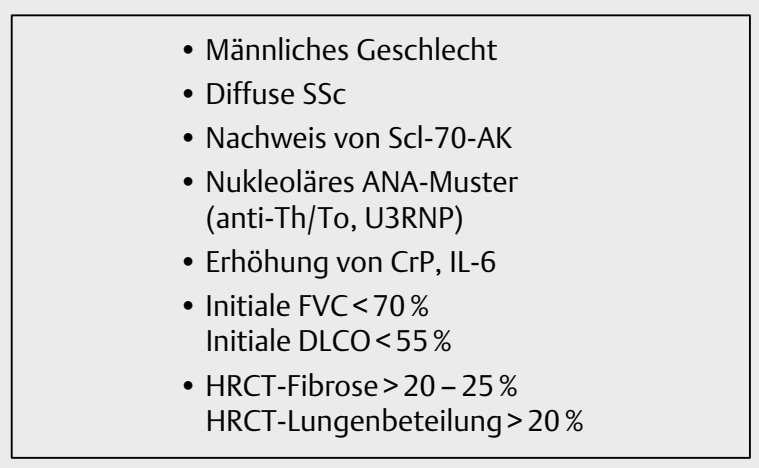

- Abb 1 Risikofaktoren für die ILD-Progression bei SSC (FVC = forcierte Vitalkapazität, DLCO = Diffusionskapazität der Lunge für Kohlenmonoxid).

lauf der ILD analysiert. Die Prognoseeinschätzung bzw. Risikostratifizierung beruht lediglich auf 2 Parametern, dem Ausmaß der interstitiellen Veränderungen im HRCT und der FVC. Für die initiale Analyse wurde zunächst eine Unterteilung basierend auf dem Ausmaß (Angabe in \% des Lungenvolumens) der interstitiellen Veränderungen im HRCT vorgenommen. Patienten mit einem Ausmaß $<10 \%$ wurden als limitierte, jene mit $>30 \%$ als extensive ILD eingeordnet. Bei intermediärem Befund im HRCT erfolgte die weitere Zuordnung in Abhängigkeit von der FVC, bei FVC $\geq 70 \%$ in die Gruppe der limitierten ILD, bei FVC $<70 \%$ als extensive ILD. Für den Tod der Patienten nach 120 Monaten waren folgende initiale Befunde bzw. Befundkonstellationen prädiktiv: Einordnung als limitierte oder extensive ILD unter Kombination von Bildgebung und FVC (HR 3,46; $p<0,0005)$, FVC $\geq 70 \%$ bzw. $<70 \%$, (HR 2,11; $p=0,001)$, Ausmaß der interstitiellen Veränderungen im HRCT $\leq$ bzw. $>20 \%$ (HR 2,48; p<0,0005). Für die klinische Praxis wird folgende etwas vereinfachte Unterteilung in limitierte und extensive ILD vorgeschlagen: Die limitierte ILD ist gekennzeichnet durch ein Ausmaß der interstitiellen (retikulären) Veränderungen im HRCT von eindeutig weniger als $20 \%$ des Lungenvolumens, von einer extensiven ILD wird gesprochen, wenn eindeutig mehr als $20 \%$ der Lunge von den interstitiellen Veränderungen betroffen sind. Ist das Ausmaß der Veränderungen in der HRCT nicht eindeutig zuzuordnen (intermediärer Befund) wird die ILD bei einer FVC von $\geq 70 \%$ der limitierten, bei einer FVC von $<70 \%$ der extensiven ILD zugeordnet.

Ähnlichen Prämissen folgt eine 2019 vorgeschlagene Einteilung der SSc-ILD in eine subklinische und klinische ILD [9]. Diese Unterteilung zielt vordergründig auf eine Differenzierung der Patienten, bei denen engmaschige Kontrollen von Lungenfunktion und ggf. der Bildgebung ausreichend sind und zunächst auf eine immunsuppressive Therapie verzichtet werden kann, von jenen bei denen eine umgehende Immunsuppression indiziert ist. Die subklinische ILD ist nach dieser Einteilung gekennzeichnet durch fehlende ILDSymptome, minimale bis milde Veränderungen im HRCT, eine normale Lungenfunktion (FVC und DLCO) und das Fehlen einer klinisch bedeutsamen Verschlechterung von FVC und/oder DCLO. Für die Einordung als subklinische ILD müssen alle der genannten Merk- 


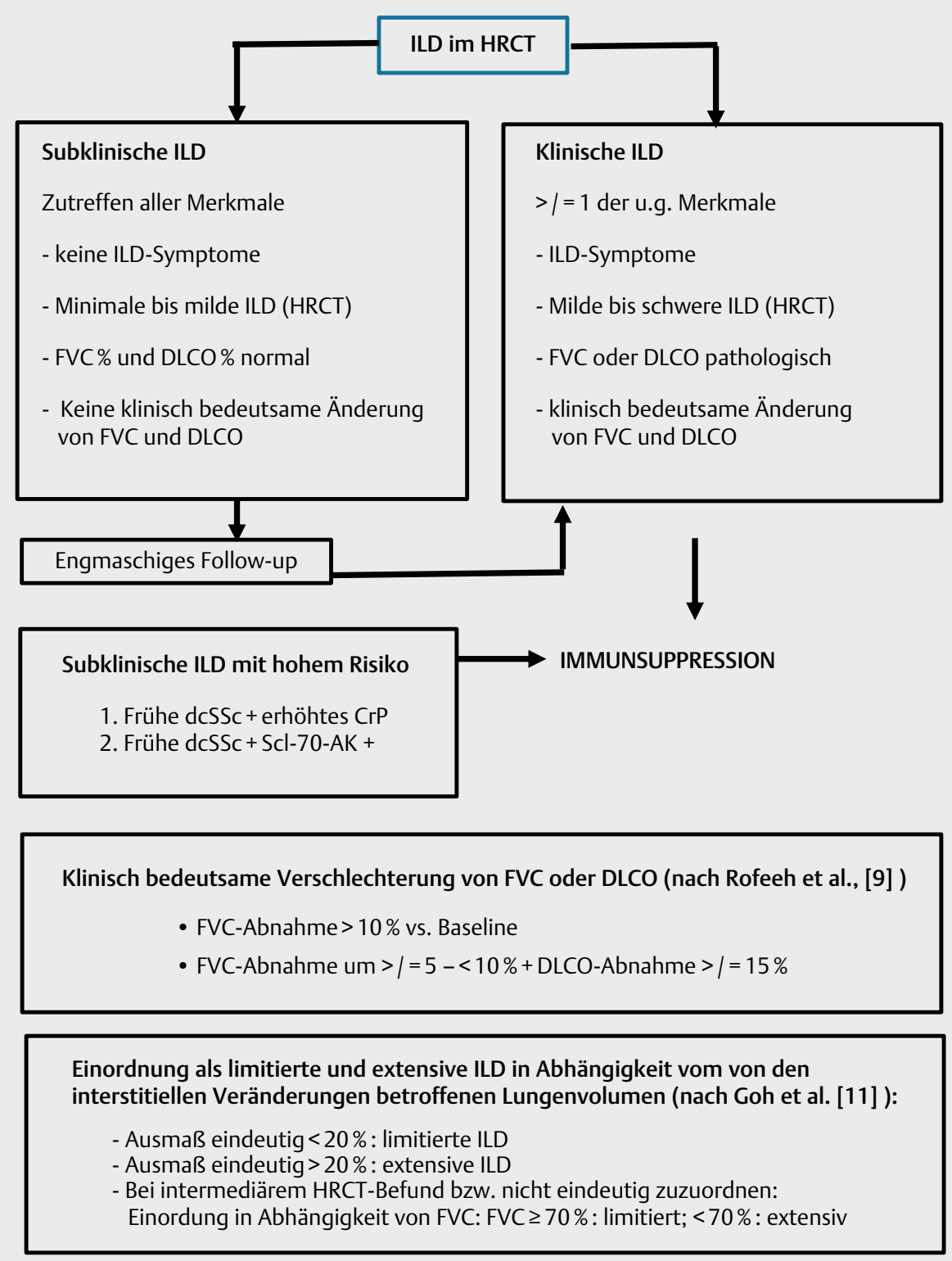

- Abb. 2 Risikobasierter Algorithmus für Monitoring und Therapieindikation bei SSc-ILD (FVC = forcierte Vitalkapazität; DLCO = Diffussionskapazität der Lunge für Kohlenmonoxid, dcSSc= diffuse cutane Sklerodermie).

male zutreffen. Als klinische ILD werden jene Patienten eingeordnet, für die mindestens eins der folgenden Kriterien zutrifft: Milde bis schwere Veränderungen im HRCT, reduzierte Werte für FVC und/oder DLCO, klinisch bedeutsame Verschlechterung von FVC und/oder DLCO im Verlauf bzw. Vorhandensein von ILD-Symptomen. Als klinisch relevante Verschlechterung der Lungenfunktion werden gewertet: Eine Abnahme der FVC um $>10 \%$ und/oder eine Abnahme der FVC um $\geq 5-<10 \%$ in Kombination mit einer Abnahme der DLCO um $\geq 15 \%$. Ein Risiko-basierter Algorithmus für Monitoring und Therapieindikation bei SSc-ILD zeigt $>$ Abb. 2. Im Falle einer Immunsuppression kommen nach den aktuellen Empfehlungen und der Studienlage primär Cyclophosphamid oder Mycophenolatmofetil (MMF) als Induktionstherapie, ggf. auch Rituximab in Betracht [1]. Die Zulassung von Nintedanib als Fibrose-hemmen- des Therapieprinzip eröffnet neue therapeutische Möglichkeiten, wobei hier eine Kombination mit einer Immunsuppression mit MMF einen zusätzlichen Nutzen im Hinblick auf die Hemmung der Progredienz der ILD zeigt [12].

\section{Pulmonal-arterielle Hypertonie bei SSc}

Im Vergleich zur ILD sind mit 6-12\% weniger Patienten mit SSc von einer pulmonalen Hypertonie (PH) betroffen $[13,14]$. Eine Metaanalyse fünf europäischer Studien mit 1165 SSc-Patienten zeigte für die PH eine Prävalenz von 7 \% [14]. Basierend auf Klinik und Pathophysiologie wird die PH in 5 Gruppen unterteilt. Gruppe 1: Pulmonal-arterielle Hypertonie (PAH), Gruppe 2: PH bei Linksherzerkrankungen, Gruppe 3: PH bei Lungenerkrankungen und/oder Hypoxie, Gruppe 4: chronische thromboembolische pulmonale 
Hypertonie (CTEPH) und Gruppe 5: PH unklarer oder multifaktorieller Ursache [15]. In o.g. Metaanalyse hatten $77 \%$ der von $\mathrm{PH}$ betroffenen SSc-Patienten eine präkapilläre $\mathrm{PH}$, davon gehörten 2/3 zur Gruppe 1 (PAH) und 1/3 zur Gruppe 3 (sekundäre PH bei interstitieller Lungenerkrankung) [13]. Bezogen auf die gesamte Patientengruppe mit PH gehörten 51 \% zur Gruppe 1, 26 \% zur Gruppe 3, 21 \% hatten eine PH bei Linksherzerkrankung (Gruppe 2) und $2 \%$ eine $\mathrm{PH}$ bei pulmonal vascular occlusive disease (PVOD). Eine ähnliche Häufigkeitsverteilung fand sich in der DETECT-Studie mit $60 \% \mathrm{PAH}$ und $40 \% \mathrm{PH}$ der anderen o.g. Gruppen [16].

Die Diagnose der PAH wird mittels Rechtsherzkatheterisierung gestellt, wobei eine PAH definiert ist als mittlerer pulmonal-arterieller Druck (mPAP) von $\geq 25 \mathrm{mmHg}$ bei einem pulmonal-kapillären Verschlußdruck von $\leq 15 \mathrm{mmHg}[15,16]$. Im Rahmen einer modifizierten Definition wurden 2019 bereits ein mPAP> $20 \mathrm{mmHg}$ und ein pulmonal-vaskulärer Widerstand $\geq 3$ Wood units als PAH definiert [13]. Um bei o.g. Prävalenz eine sinnvolle Differenzialindikation für die Rechtsherzkatheterisierung zu stellen, ist neben regelmäßigen echokardiografischen Kontrollen aller SSc-Patienten auf Zeichen einer PH die Kenntnis der Risikofaktoren für eine PAH essenziell. Als einfach zu ermittelnde Risikofaktoren für eine PAH gelten bestimmte demografische Faktoren wie eine lange bestehende Erkrankung, hohes Lebensalter bei Beginn der SSc und eine lange postmenopausale Zeitspanne [17]. Ferner signalisieren klinische Befunde welche das Ausmaß der obstruktiven Vaskulopathie und das pathologische Gefässremodeling erkennen lassen, ein erhöhtes Risiko für eine PAH. Dazu zählen eine schwere RaynaudSymptomatik, schwere digitale Ischämien sowie das Vorhandensein von Teleangiektasien [17]. Desweiteren sind der Nachweis von Centromer-AK und eine Hyperurikämie mit einem höheres PAHRisiko assoziiert [16]. Ferner konnte nachgewiesen werden, daß stimulierende Antikörper gegen Endothelin-A-Rezeptor und Angiotensin-1-Rezeptor prädiktiv für die Entwicklung einer PAH bei SSc sind und mit einer erhöhten Mortalität einhergehen [18]. Befunde der Lungenfunktion welche auf eine PAH hindeuten, sind eine inadäquat niedrige DLCO bei relativ guter FVC, welche sich in einer FVC/DLCO-Ratio von > 1,6 widerspiegelt bzw. eine isolierte Verringerung der DLCO auf< $50 \%$ [17]. Risikofaktoren für eine PAH bzw. mit PAH assoziierte Befunde sind in $>$ Tab. 1 dargestellt. Viele der genannten Parameter haben in den DETECT-Algorithmus zur
Frühdiagnose der PAH bei SSc Eingang gefunden [16]. Im ersten Schritt des DETECT-Algorithmus werden die FVC\%/DLCO \%-Ratio, das aktuelle oder frühere Vorhandensein von Teleangiektasien, das Vorhandensein von Centromer-AK, das Serum NTproBNP, die Serumharnsäure und das Vorhandensein eines überdrehten Rechtstyps im EKG zur Ermittlung eines Gesamtrisiko-Index herangezogen [16]. Liegt dieser Index > 300, wird die Indikation zur Echokardiografie gestellt und in einem zweiten Schritt der rechte Vorhof und die Trikuspidalregurgitation beurteilt. Liegt der nun ermittelte Gesamt-Risiko-Index bei > 35 wird die Indikation zur invasiven Diagnostik mittels Rechtsherzkatheter gestellt. In einem entsprechenden Modell wird gezeigt, daß durch Verwendung des DETECTAlgorithmus gegenüber dem von der ESC/ERS empfohlenen Vorgehen bei SSc die Sensitivität für die Diagnose der PAH von 71 auf $96 \%$ und der negative Vorhersagewert von 89 auf $89 \%$ gesteigert werden kann. Infolgedessen wurde die Zahl der nicht diagnostizierten Patienten mit PAH von 29 auf $4 \%$ reduziert [16].

Bei diagnostizierter PAH ist zur Etablierung einer gezielten Differenzialtherapie mit dem Ziel einer Verbesserung der Prognose eine Risikostratifizierung des Schweregrades der PAH entscheidend, der die prognostizierte Einjahresmortalität zugrunde gelegt wird [15]. Dabei dient diese Risikostratifizierung insbesondere auch einer frühzeitigen Identifizierung von Patienten, welche bereits initial oder in Abhängigkeit vom Verlauf einer Kombinationstherapie zugeführt werden sollten. Eine Einstufung als niedriges Risiko erfolgt bei einer 1 -Jahres-Mortalität von < $5 \%$, als intermediäres Risiko bei einer 1-Jahres-Mortalität von 5-10\% und als hohes Risiko bei einer 1-Jahres-Mortalität von > 10\%. Als Determinanten des Risikos bzw. der Prognose konnten verschiedene anamnestische und klinische (Symptomprogression, Auftreten von Synkopen, Vorhandensen klinischer Zeichen einer Rechtsherzinsuffizienz, funktionelle WHO-Klasse), funktionsdiagnostische (Ergebnisse des 6-Minuten-Gehtests und des kardiopulmonalen Belastungstests), laborchemische (BNP bzw. NTproBNP), echokardiografische (Fläche des rechten Vorhofs, Vorhandensein bzw. Ausmaß eines möglichen Perikardergusses) sowie hämodynamische (rechtsatrialer Druck, Cardiac Index, gemischt-venöse Sauerstoffsättigung) Befunde identifiziert werden. Die Einschätzung des Risikos ist somit immer eine interdisziplinäre Leistung. Andererseits kann ein Teil der Risikoeinschätzung durch jeden Arzt anhand einfacher klini-

- Tab. 1 Klinische, funktionsdiagnostische und serologische Risikofaktoren für die Entwicklung einer pulmonal-arteriellen Hypertonie (PAH) bei SSc.

\begin{tabular}{|c|c|c|}
\hline Demografische und klinische Risikofaktoren & Risikofaktoren aus der Funktionsdiagnostik & Serologische Risikofaktoren (Autoantikörper) \\
\hline $\begin{array}{l}\text { - Lange Erkrankungsdauer } \\
\text { - Beginn der SSc im höheren Lebensalter } \\
\text { - Postmenopause } \\
\text { - Schweres Raynaud-Syndrom } \\
\text { - Schwere digitale Ischämie } \\
\text { - Cutane Teleangiektasien }\end{array}$ & $\begin{array}{l}\text {. } \text { DLCO }<50 \% \text { (isoliert) } \\
\text { " } \text { DLCO/VA }<70 \% \\
\text {. FVC/DLCO }>1,6 \\
\text { - Anstieg des RSVP } \\
>2 \mathrm{mmHg} / \mathrm{Jahr}\end{array}$ & $\begin{array}{l}\text { " Zentromer-AK } \\
\text { - U1-RNP-AK } \\
\text { " Nukleoläres ANA-Muster (anti-Th/To-AK; ant } \\
\text { U3-RNP-AK, anti-B23-AK) } \\
\text { " aPL-AK } \\
\text { - AECA } \\
\text { - Anti-AT1R-AK } \\
\text { - } \text { Anti-ETAR-AK }\end{array}$ \\
\hline
\end{tabular}

DLCO = Diffussionskapazität; DLCO/VA = Diffusionskapazität bezogen auf das alveloare Volumen; FVC = forcierte Vitalkapazität; $R$ VSP= Rechtsventrikulärer systolischer Druck, RNP= Ribonukleoprotein; aPL-AK = Antiphospholipid-AK; AECA=Anti-Endothelzell-AK; Anti-AT1R-AK=stimulierende AK gegen Angiotensin-1-Rezeptor; Anti-ETAR-AK = stimulierende AK gegen Endothelin-1-Rezeptor. 
scher Parameter und Tests erfolgen. So sprechen das Fehlen von Symptomprogression, Zeichen der Rechtsherzinsuffizienz und Synkopen, eine WHO-Klasse I-II sowie ein 6-Minuten-Geh-Test von $>440$ m für ein geringes Risiko, rasche Symptomprogression, klinische Zeichen der Rechtsherzinsuffizienz, rezidivierende Synkopen, eine WHO-Klasse IV sowie ein 6-Minuten-Geh-Test von < 165 m für ein hohes Risiko bzw. für eine 1-Jahres-Mortalität von > $10 \%$. Passend zu einem intermediären Risiko sind eine langsame Symptomprogression, gelegentliche Synkopen und eine WHO-Klasse III bei fehlenden Zeichen einer Rechtsherzinsuffizienz sowie ein 6-Minuten-Gehtest von 165-440 m. Auch laborchemische Parameter zur Risikostratifizierung können leicht erhoben werden. Charakteristisch für ein niedriges Risiko sind ein BNP von $<50$ bzw. ein NTproBNP von $<300$ pg/ml, für ein hohes Risiko sprechen ein BNP von $>300$ bzw. ein NTproBNP von > 1400 pg/ml, entsprechende Werte dazwischen für ein intermediäres Risiko. Wichtige Parameter zur Risikostratifizierung bei PAH sind in den - Tab. 2 und $\mathbf{3}$ dargestellt. Wichtig ist der Hinweis, daß die vorgeschlagenen Variablen und die jeweiligen Grenzwerte in der Regel auf Expertenmeinungen basieren und in erster Linie bei der idiopathischen PAH validiert wurden [15]. Eine Übertragbarkeit auf andere Formen der $\mathrm{PAH}$ ist somit nicht zwingend gegeben.

Die Risikostratifizierung bei PAH ist sowohl für die therapeutische Strategie als auch zur Beurteilung des Therapieerfolges von grundlegender Bedeutung. Es ist in diesem Zusammenhang wichtig darauf hinzuweisen, daß die u.g. therapeutischen Ansätze für die PAH entwickelt wurden und gelten, welche grundsätzlich von der der pulmonalen Hypertonie bei ILD abgegrenzt werden muss. Bezüglich der aktuell addressierten Signalwege in der Therapie der $\mathrm{PAH}$ (Prostacyclin pathway, Endothelin pathway und NO pathway) muss zwischen Monotherapie sowie sequentieller und initialer Kombinationstherapie entschieden werden. Basierend auf den $\mathrm{Er}$ gebnissen verschiedener Studien zum Vergleich von Mono- und Kombinationstherapie geht der Trend eindeutig zur frühen Kombinationstherapie. Spätestens ab WHO-Klasse III und intermediärem Risiko sollte eine primäre Kombinationstherapie erfolgen. Bei WHO-Klasse II und geringem Risiko kann auch primär eine Monotherapie erfolgen, welche aber bei unzureichendem Effekt nach 3 Monaten zu einer sequenziellen Kombinationstherapie eskaliert werden sollte [19]. Das Ziel der PAH-Therapie besteht darin, den
Patienten in einem Niedrigrisikoprofil zu halten bzw. dahin zu überführen.

\section{Renale Krise bei SSc}

Auch die renale Krise zählt mit einer Häufigkeit von 11 \% bei dcSSc und $4 \%$ bei limitierter cutaner SSc zu den relativ seltenen, aber schweren Manifestationen der SSc [20-23]. Als Risikofaktoren einer renalen Krise gelten das Vorliegen einer dcSSc, höheres Alter, männliches Geschlecht, Perikarderguss, Gelenkkontrakturen, vorbestehende Proteinurie, genetische Faktoren wie der Nachweis von HLA-DRB1 * 0407 und HLA-DRB1 * 1304, Einnahme von Glukokortikoiden in Dosen von > 15 mg Prednisolonäquivalent pro Tag, Therapie mit Cyclosporin, der Nachweis RNA-Polymerase III-Antikörpern sowie eines gesprenkelten ANA-Musters in der Immunfluoreszenz [20-23], - Tab. 4. Obwohl ACE-Hemmer (ACE-I) die wesentliche Grundlage der Therapie der renalen Krise bilden, konnte auf Basis einer EUSTAR-Analyse von 14524 SSc-Patienten eine vorbestehende ACE-I-Therapie als unabhängiger Risikofaktor für eine renale Krise identifiziert werden [23]. Risikofaktoren einer ungünstigen Prognose der renalen Krise sind höheres Alter, männliches Geschlecht, ungenügend kontrollierter Blutdruck, Herzinsuffizienz, initial erhöhte Kreatininwerte, Therapie mit Glukokortikoiden, Vortherapie mit ACE-I und Notwendigkeit einer Dialyse [20].

\section{Kardiale Beteiligung bei SSC}

Die Prävalenz kardialer Manifestationen bei SSc wird mit 7-44\% angegeben. Die große Variabilität ist auf die unterschiedliche Intensität der kardialen Diagnostik und die differenten zum Einsatz kommenden diagnostischen Verfahren (Echokardiografie, Langzeit-EKG, Kardio-MRT, SPECT, PET, Bestimmung natriuretischer Peptide) zurückzuführen [24]. Häufig beschrieben werden linksventrikuläre diastolische Dysfunktion (17,7-51,9\%), primäre rechtsventrikuläre Dysfunktion (38\%), mikrovaskuläre koronare Herzerkrankung (>60\%), Perikardergüsse (15-72\%) sowie Tachyarrhythmien (17-28\%). Linksventrikuläre Dysfunktion (1,4-7\%) und Perikarditis (1,9-9\%) sind seltener, auch eine Myokarditis wird selten gefunden [24]. Eine Analyse mit 12829 Patienten aus 8 Studien zeigte für SSc-Patienten mit kardialer Beteiligung eine erhöhte Mortalität (HR 3,15) [25]. Risikofaktoren für die Entwicklung einer kardialen Manifestation wurden in der EUSTAR-Kohorte mit früher SSc untersucht [26]. Ein Jahr nach

- Tab. 2 Risikostratifizierung basierend auf der erwarteten 1-Jahres-Mortalität bei pulmonal-arterieller Hypertonie (PAH): Klinische und laborchemische Parameter (Niedriges Risiko: 1-Jahres-Mortalität<5\%; Intermediäres Risiko: 1-Jahres-Mortalität 5-10\%; Hohes Risiko: 1-Jahres-Mortalität $>10 \%$ ) (nach [15] - ESC/ERS guidelines).

\begin{tabular}{|c|c|c|c|}
\hline \multirow[b]{2}{*}{ Prognosefaktoren } & \multicolumn{3}{|c|}{ Risiko } \\
\hline & Niedrig $<5 \%$ & Intermediär 5-10\% & Hoch $>10 \%$ \\
\hline Klinische Zeichen der Rechtsherzinsuffizienz & - & - & + \\
\hline Symptomprogredienz & - & langsam & rasch \\
\hline Synkopen & - & gelegentlich & wiederholt \\
\hline Funktionelle WHO-Klasse & I, II & III & IV \\
\hline 6-Minuten-Gehtest & $>440 \mathrm{~m}$ & $165-440 \mathrm{~m}$ & $<165 \mathrm{~m}$ \\
\hline BNP im Plasma & $<50 \mathrm{ng} / \mathrm{l}$ & $50-300 \mathrm{ng} / \mathrm{l}$ & $>300 \mathrm{ng} / \mathrm{l}$ \\
\hline NT-proBNP im Plasma & $<300 \mathrm{ng} / \mathrm{l}$ & $300-1400 \mathrm{ng} / \mathrm{l}$ & $>1400 \mathrm{ng} / \mathrm{l}$ \\
\hline
\end{tabular}


- Tab. 3 Risikostratifizierung basierend auf der erwarteten 1-Jahres-Mortalität bei pulmonal-arterieller Hypertonie (PAH): Funktionsdiagnostische, echokardiografische und hämodynamische Parameter (Niedriges Risiko: 1-Jahres-Mortalität< 5\%; Intermediäres Risiko: 1-Jahres-Mortalität 5-10\%; Hohes Risiko: 1-Jahres-Mortalität>10\%) (nach [15] - ESC/ERS guidelines).

\begin{tabular}{|c|c|c|c|}
\hline & \multicolumn{3}{|c|}{ Risiko } \\
\hline Prognosefaktoren & Niedrig $<5 \%$ & Intermediär 5-10\% & Hoch $>10 \%$ \\
\hline Kardiopulmonaler Belastungstest & $\begin{array}{l}\text { Peak VO2 }>15 \mathrm{ml} / \mathrm{min} . / \mathrm{kg}(>65 \%) \\
\text { VE/CO2-Anstieg }<36\end{array}$ & $\begin{array}{l}\text { Peak VO2 11-15 ml/min./kg (35-65\%) } \\
\text { VE/CO2-Anstieg <36-44,9 }\end{array}$ & $\begin{array}{l}\text { Peak VO2<11 } \mathrm{ml} / \mathrm{min} . / \mathrm{kg}(<35 \%) \\
\text { VE/CO2-Anstieg } \geq 45\end{array}$ \\
\hline $\begin{array}{l}\text { Bildgebung. Echokardiografie } \\
\text { Kardio-MRT: RA-Fläche }\end{array}$ & $<18 \mathrm{~cm} 2$ & $18-26 \mathrm{~cm} 2$ & $>26 \mathrm{~cm} 2$ \\
\hline $\begin{array}{l}\text { Bildgebung. Echokardiografie } \\
\text { Kardio-MRT: Perikarderguss }\end{array}$ & - & - bis minimal & + \\
\hline Hämodynamische Parameter & $\begin{array}{l}\mathrm{RAP}<8 \mathrm{mmHg}, \mathrm{Cl} \geq 2,5 \mathrm{l} / \mathrm{min} / \mathrm{m} 2, \\
\mathrm{SVO} 2>65 \%\end{array}$ & $\begin{array}{l}\text { RAP 8-14 mmHg, Cl 2,0-2,4 I/min/m2, } \\
\text { SvO2 60-65\% }\end{array}$ & $\begin{array}{l}\text { RAP }>14 \mathrm{mmHg}, \mathrm{Cl}<2,0 \mathrm{l} / \mathrm{min} / \mathrm{m} 2, \\
\mathrm{SVO} 2<60 \%\end{array}$ \\
\hline
\end{tabular}

Peak $\mathrm{VO}_{2}=$ maximaler Sauerstoffverbrauch; $\mathrm{VE} / \mathrm{CO}_{2}=$ Anstieg der ventilatorischen Äquivalente für Kohlendioxid; $\mathrm{RA}=$ rechter Vorhof; $\mathrm{RAP}=$ rechtsatrialer Druck; $\mathrm{Cl}=$ Cardiac Index; $\mathrm{SvO}_{2}=$ gemischt venöse Sauerstoffsättigung Peak, $\mathrm{VO}_{2}=$ maximaler Sauerstoffverbrauch; $\mathrm{VE} / \mathrm{CO}_{2}=$ Anstieg der ventilatorischen Äquivalente für Kohlendioxid; RA= rechter Vorhof.

- Tab. 4 Renale Krise bei SSc: Risikofaktoren und assoziierte Faktoren (dcSSc = diffuse cutane Sklerodermie).

\begin{tabular}{|l|l|}
\hline \multirow{2}{*}{ Demographische Faktoren } & Höheres Lebensalter \\
\hline \multirow{2}{*}{ Klinische Befunde } & Männliches Geschlecht \\
\hline & dcSSc \\
\hline & Perikarderguss \\
\hline Gelenkkontrakturen \\
\hline Laborchemische Befunde & Vorbestehende Proteinurie \\
\hline Serologische Befunde & $\begin{array}{l}\text { RNA-Polymerase III-Antikörper, } \\
\text { gesprenkeltes ANA-Muster }\end{array}$ \\
\hline Genetische Faktoren & HLA-DRB1 ${ }^{*}$ 0407, HLA-DRB1 ${ }^{*} 1304$ \\
\hline Vortherapie & $\begin{array}{l}\text { Glukokortikoide>15 mg } \\
\text { Prednisolonäquivalent/d }\end{array}$ \\
\hline & ACE-Hemmer \\
\hline Cyclosporin A
\end{tabular}

Beginn der Raynaud-Symptomatik fand sich bei 695 Patienten mit früher SSc in 32 \% eine kardiale Beteiligung. Als Risikofaktoren für eine kardiale Beteiligung konnten höheres Lebensalter (2,1-fach höhere Inzidenz), dcSSc (1,9-fach höhere Inzidenz im Vergleich zu IcSSc) sowie der Nachweis von Topoisomerase I-Antikörpern im Vergleich zu Patienten mit Anticentromer-Antikörpern (3,7-fach höhere Inzidenz) herausgearbeitet werden. In der multivariaten Analyse waren höheres Lebensalter (HR 1,04 pro um 1 Jahr höheres Alter) und Topoisomerase I-Antikörper (HR 3,9) Risikofaktoren für die Entwicklung einer kardialen Beteiligung. Die häufigste kardiale Manifestation war eine diastolische Dysfunktion mit 15\% gefolgt von Erregungsüberleitungsstörungen (8,9\%) und Perikardergüssen (8,4\%). Im weiteren Verlauf hatten nach 10 Jahren ca. $60 \%$ der Patienten diastolische Dysfunktion entwickelt und mehr als 25\% Erregungsüberleitungsstörungen. Risikofaktoren für eine diastolische Dysfunktion waren höheres Lebensalter und dcSSc (in der multivariaten Analyse lediglich höheres Lebensalter), wesentlicher Risikofaktor für eine Erregungsüberleitungsstörung war der Nachweis von Topoisomerase I-Antikörpern mit einer 10-fach höheren Inzidenz im Vergleich zu Patienten mit Centromerantikörpern. Perikardergüsse entwickelten v. a. Patienten mit dcSSc (5,6-fach höhere Inzidenz im Vergleich zu IcSSc) sowie Männer (2,7-fach höhere Inzidenz im Vergleich zu Frauen). Auch kardiale Biomarker sind für die Risikostratifizierung im Hinblick auf kardiale Affektionen bei SSc geeignet. Eine prospektive Studie bei 245 Patienten mit SSc zeigte für die kardialen Biomarker hsCTNT sowie NT-proBNP bei 32,3 bzw. 31,8\% der Patienten erhöhte Werte. Erhöhte Werte dieser kardialen Biomarker waren assoziiert mit diffusem Hautbefall, hohem Rodnan Skin Score, reduzierter linksventrikulärer Ejektionsfraktion, Auftreten eines Rechtsschenkelblocks sowie erhöhter Mortalität [27].

\section{Digitale Ulcerationen bei SSc}

Aufgrund der durch Schmerzen, Funktionsverlust, Arbeitsunfähigkeit und häufige Hospitalisation bedingten Reduktion der Lebensqualität zählen digitale Ulcerationen (DU) zu den klinisch relevantesten Manifestationen der SSc. Verschiedene Analysen zeigten, daß sowohl demographische Faktoren als auch klinische Ausprägung bzw. Manifestationen der SSc sowie Autoantikörper mit der Entwicklung von DU assoziiert und somit zur Risikostratifizierung geeignet sind. In der Analyse einer australischen Arbeitsgruppe, welche 1085 SSc-Patienten umfaßte, entwickelten 48,6\% der Patienten über einen mittleren Beobachtungszeitraum von 5,2 Jahren DU [28]. Patienten mit DU waren gegenüber jenen ohne DU bei Erkrankungsbeginn im Mittel jünger ( $p<0,001)$, hatten eine längere Erkrankungsdauer ( $p=0,001)$ und waren häufiger männlichen Geschlechts ( $p=0,002)$. Ferner zeigte sich für Patienten mit DU eine Assoziation mit folgendenen klinischen Befunden bzw. Erkrankungsmanifestationen: Diffuse cutane SSc $(p<0,001)$, Vorhandensein von Teleangiektasien $(p<0,001)$, Calcinose $(p<0,001)$, Gelenkkontrakturen ( $p<0,001)$, gastrointestinaler Beteiligung $(p<0,001)$, ILD $(p=0,003)$ und PAH $(p=0,02)$. Patienten mit DU wiesen häufiger Topoisomerase I-Antikörper $(p<0,001)$ und RNA- 
Polymerase III-Antikörper ( $p=0,05)$ auf und hatten seltener Centromer-Antikörper $(p=0,007)$. Auch in einer Analyse der EUSTARDatenbank mit 3656 Patienten zeigte sich eine Assoziation von DU mit dcSSc und Topoisomerase-I-Antikörpern [29]. Außerdem fand sich sowohl bei dcSSc als auch bei IcSSc eine Assoziation von DU mit früh im Krankheitsverlauf auftretender Raynaud-Symptomatik [29]. Zu sehr ähnlichen Ergebnissen kam eine Untersuchung aus Deutschland mit 1881 Patienten [30]. In der multivariaten Analyse zeigten männliches Geschlecht, PAH, Ösophagusbeteiligung, dcSSc, Topoisomerase I-Antikörper, junges Alter bei Beginn der Raynaud-Symptomatik sowie erhöhte BSG eine signifikante Assoziation mit DU. Topoisomerase I-AK-positive Männer mit früher Raynaud-Symptomatik, $\mathrm{PAH}$ und einer BSG von $>30 \mathrm{~mm} / \mathrm{h}$ wiesen mit 88 \% am häufigsten DU auf. Die Assoziation von DU mit bestimmten klinischen Manifestationen wie dcSSc, ILD inklusive reduzierter Diffussionskapazität, Oesophagusbeteiligung, jüngerem Alter bei Krankheitsbeginn, längerer Erkrankungsdauer sowie mit Topoisomerase-I-Antikörpern konnte auch in einer kanadischen SSc-Kohorte mit 938 Patienten bestätigt werden [31]. Ferner fand sich eine hochsignifikante Assoziation mit einem hohen modifizierten Rodnan Skin Score und dem Skin-Score von Hand und Fingern. In einer Übersicht, welche 13 Studien zu Risikofaktoren für DU erfaßt, wurden dcSSc und Nachweis von Topoisomerase I-Antikörpern in jeweils 9 Studien, frühe SSc-Symptome außerhalb des RaynaudSyndroms in 6 Studien sowie frühes Raynaud-Syndrom, hoher Rodnan Skin Score sowie männliches Geschlecht in jeweils 5 Studien als Risikofaktoren für DU genannt [32]. Auch ein mit einer fortgeschrittenen SSc zu vereinbarendes Muster in der Kapillarmikroskopie mit vermehrtem Auftreten von Kapillarlücken sowie hohe Endothelin-1-Serumspiegel sowie niedrige Serumspiegel von VEGF sind Prädiktoren für die Entwicklung von DU. Die Beachtung der Risikofaktoren für DU kann helfen, die entsprechenden Risikogruppen frühzeitig zu analysieren und mit einer adäquaten vasoaktiven Therapie mit Calciumantagonisten, Prostanoiden, PDE-5-Inhibitoren und Bosentan entsprechend den Therapieempfehlungen und dem Zulassungsstatus zu versorgen [33].

\section{Teil 2: Risikostratifizierung bei Lupus-Nephritis}

Die Lupus-Nephritis (LN) ist mit einer von Ethnizität, Geschlecht und Alter bestimmten Prävalenz von 35-60\% eine der häufigsten und die wichtigste Prognose-bestimmende Organmanifestation des systemischen Lupus erythematodes (SLE) [34, 35]. Trotz Verbesserung der Prognose entwickeln ca. $10 \%$ der betroffenen Patienten innerhalb von 5 Jahren und bis zu $20 \%$ innerhalb von 10 Jahren eine terminale Niereninsuffizienz [34, 35]. In einer Analyse bei 635 SLE-Patienten von denen 15,3\% innerhalb von 5,7 Jahren verstarben, war die renale Domäne des SLICC Damage Index (SDI) die 19,8 \% häufigste Domäne, in welcher chronische irreversible Organschäden zu konstatieren waren und es fand sich eine Assoziation der Nierenschädigung zur Mortalität [36]. Die Verhinderung einer chronischen Nierenschädigung hat somit einen wesentlichen Einfluß auf die Langzeitprognose bei SLE. Um Strategien zu entwickeln, welche geeignet sind, die Prognose der LN zu verbessern, ist die Kenntnis der Risikofaktoren einer ungünstigen Prognose der LN bzw. eine entsprechende Risikostratifizierung essenziell.

Verschiedene Analysen hatten das Ziel, Risikofaktoren einer ungünstigen Langzeit-Prognose der LN zu ermitteln. Als Outcome-
Parameter wurden die Entwicklung einer terminalen Niereninsuffizienz (ESRD) [37-46], Tod [47], die Entwicklung einer chronischen Niereninsuffizienz [45, 48-56] sowie kombinierte Endpunkte welche ESRD und Tod [57, 58] bzw. Tod, ESRD bzw. ein definiertes Ausmaß einer anhaltenden Verschlechterung bzw. Einschränkung der Nierenfunktion umfassten, [59-63] betrachtet. Andere Untersuchungen waren auf Risikofaktoren für LN-Rezidive fokussiert [64-66]. In weiteren Studien wurden Risikofaktoren für ESRD, persistierende Proteinurie und Rezidive separat untersucht [67] bzw. Risikofaktoren für das Fehlen einer anhaltenden Remission evaluiert [68].

Einer der wichtigsten Risikofaktoren für die Entwicklung einer ESRD ist eine bereits initial bestehende Nierenfunktionseinschränkung [37-39, 42-46, 50, 58]. Als weitere wichtige Risikofaktoren für ein ESRD fanden sich arterielle Hypertonie [39, 44, 45, 58, 67], ausgeprägte tubulointerstitielle Läsionen [41] bzw. ein hoher Chronizitätsindex $(\mathrm{Cl})[39,67]$ in der Nierenbiopsie, männliches Geschlecht [37, 44], Anämie [43, 53], Klasse IV-LN [37, 44, 45], das Fehlen einer Remission im Verlauf $[38,40,41]$ und eine ausgeprägte initiale Proteinurie [44] bzw. ein nephrotisches Syndrom bei membranöser LN [41].

Risikofaktoren für das Erreichen eines kombinierten Endpunktes mit Tod, ESRD bzw. einem definierten Ausmaß einer anhaltenden Verschlechterung bzw. Einschränkung der Nierenfunktion waren initial eingeschränkte Nierenfunktion [61-63], hoher $\mathrm{Cl}$ in der Nierenbiopsie [62, 63], Hypertonie [61, 62], das Ausbleiben einer Remission [61] bzw. das Nichterreichen einer kompletten Remission (CR) [59, 60].

Als Risikofaktoren für die Entwicklung einer chronischen Niereninsuffizienz wurden initiale Nierenfunktionseinschränkung [48, 50, 52, 54, 66], der Nachweis chronischer Läsionen in der Nierenbiopsie bzw. ein hoher $\mathrm{Cl}[49,54-56]$, Hypertonie [49, 50, 55], ausgeprägtere initiale Proteinurie [48, 51], männliches Geschlecht $[51,53]$ und Ausbleiben von Remission [54] bzw. kompletter Remission (CR) [52] ermittelt. Ferner wurden Klasse IV-LN [45, 50] und hoher Aktivitätsindex [49] genannt.

In einzelnen Studien waren Hypertonie, der Nachweis einer fokal-segmentalen oder fortgeschritten sklerosierenden LN [58] sowie nephrotisches Syndrom und Antiphospholpidsyndrom [57] mit einer erhöhten Mortalität assoziiert.

Rezidive der LN waren assoziiert mit Ausbleiben einer CR [65, 66] sowie der Persistenz von dsDNA-AK und Komplementverbrauch nach Remissionsinduktion [67]. Bei Nachweis einer Klasse IV-LN wurde häufiger das Ausbleiben einer über 5 Jahre anhaltenden Remission beobachtet [68].

Risikofaktoren für eine ungünstige Prognose der LN sind in - Tab. 5 dargestellt.

Andere Studien fokussieren auch oder ausschliesslich auf die Detektion von Prädiktoren eines günstigen Outcomes der LN, wie Remission im Langzeitverlauf und Überleben ohne Niereninsuffizienz [38, 42, 69-77]. Weitgehend übereinstimmend werden eine effektive Reduktion der Proteinurie innerhalb der ersten 12 Monate nach Beginn Remissionsinduktion auf Werte zwischen <0,5-0,8 g/d [74, 75, 77] bzw. eine $50 \%$-ige Reduktion der Proteinurie nach 6 Monaten [71] oder auch eine über 6 Monate erhaltene Reduktion der Urin-Protein/Kreatinin-Ratio (UPCR) auf $<0,3 \mathrm{~g} / \mathrm{gKreatinin}$ [76] als prädiktiv für eine gute Langzeitprognose der LN beschrie- 
- Tab. 5 Risikofaktoren für ungünstige Langzeitprognose der Lupus-Nephritis (LN); (CR=komplette Remission).

\section{Risikostratifizierung für Progression zur terminalen Niereninsuffizienz (ESRD)}

\begin{tabular}{|l|l|l|}
\hline Parameter & Prädiktoren ungünstiger Prognose & Referenzen \\
\hline Nierenfunktion & bereits initial bestehende Nierenfunktionseinschränkung & {$[37-39,42-46,50,58]$} \\
\hline Proteinurie & Hohe initiale Proteinurie nephrotisches Syndrom (bei Klasse V) & {$[41,44]$} \\
\hline Hämatologie & Anämie & {$[43,53]$} \\
\hline Komorbidität & (Schlecht kontrollierte) arterielle Hypertonie & {$[39,44,45,58,67]$} \\
\hline Demographische Faktoren & männliches Geschlecht & {$[37,44]$} \\
\hline Nierenhistologie & ausgeprägte tubulointerstitielle Läsionen & {$[41]$} \\
\hline & ein hoher Chronizitätsindex (Cl) & {$[39,67]$} \\
\hline Therapieansprechen & Klasse IV-LN & {$[37,44-45]$} \\
\hline
\end{tabular}

Risikostratifizierung für Entwicklung einer chronischen Niereninsuffizienz (NI)

\begin{tabular}{|l|l|l|}
\hline Parameter & Prädiktoren ungünstiger Prognose & Referenzen \\
\hline Nierenfunktion & bereits initial bestehende Nierenfunktionseinschränkung & {$[48,50,52,54,66]$} \\
\hline Proteinurie & Ausgeprägtere initiale Proteinurie & {$[48,51]$} \\
\hline Komorbidität & (Schlecht kontrollierte) arterielle Hypertonie & {$[49,50,55]$} \\
\hline Demographische Faktoren & männliches Geschlecht & {$[51,53]$} \\
\hline Nierenhistologie & Nachweis chronischer Läsionen in der Nierenbiopsie bzw. hoher Cl & {$[49,54-56,66]$} \\
\hline & Hoher Aktivitätsindex & {$[49]$} \\
\hline Therapieansprechen & Klasse IV-LN & {$[45,50]$} \\
\hline & Ausbleiben von Remission bzw. CR & {$[52,54]$} \\
\hline
\end{tabular}

Risikostratifizierung: kombinierter Endpunkt (Tod u/o. ESRD, anders definierte NI)

\begin{tabular}{|l|l|l|}
\hline Parameter & Prädiktoren ungünstiger Prognose & Referenzen \\
\hline Nierenfunktion & bereits initial bestehende Nierenfunktionseinschränkung & {$[61-63]$} \\
\hline Komorbidität & (Schlecht kontrollierte) arterielle Hypertonie & {$[61-62]$} \\
\hline Nierenhistologie & hoher Cl & {$[62-63]$} \\
\hline Therapieansprechen & Ausbleiben von Remission bzw. CR & {$[59-61]$} \\
\hline
\end{tabular}

ben. In 3 Analysen war ein niedriger $\mathrm{Cl}$ in der Nierenbiopsie mit einer guten Langzeitprognose assoziiert $[38,69,73]$.

Für die Risikostratifizierung bei LN läßt sich somit schlußfolgern, daß v. a. eine bereits initial bestehende Niereninsuffizienz sowie chronische irreversible Gewebsschäden in der Niere, welche in einem hohen $\mathrm{Cl}$ in der Nierenbiopsie zum Ausdruck kommen und einer Immunsuppression nicht zugänglich sind, offenbar die größte Bedeutung für eine schlechte Prognose haben, ibs. im Hinblick auf die Entwicklung einer chronischen Niereninsuffizienz und einer ESRD. Interessanterweise wurde in drei Studien auf die Bedeutung der Verzögerung von Diagnose der LN bzw. therapeutischer Intervention für eine schlechte Prognose der LN hingewiesen $[52,58,64]$. Weitere wichtige ungünstige Prognosefaktoren sind Hypertonie, männliches Geschlecht und das Ausbleiben einer (kompletten) Remission. Die initiale Aktivität der LN sowie die histologische Klassifizierung sind dagegen offenbar von geringerer Bedeu- tung. Zwar findet sich eine Assoziation zwischen einer Klasse IV-LN mit ungünstiger Prognose in 5 Studien [37, 44, 47, 50, 67]. Jedoch wird eine Beziehung zwischen einem hohen Aktivitätsindex in der Nierenbiopsie und einer ungünstigen Entwicklung der Nierenfunktion nur in zwei älteren Studien $[49,57]$ beschrieben. Auch für immunologische Parameter wie den Nachweis bestimmter Auto-Antikörper sowie Komplementverbrauch fand sich nur in 4 Studien $[38,55,61,67]$ eine prognostische Bedeutung im Hinblick auf eine ungünstige Entwicklung der renalen Funktion. Der effektiven Reduktion der Proteinurie im Rahmen der Induktionstherapie kommt offenbar eine entscheidende Bedeutung im Hinblick auf die Langzeitprognose der LN zu.

Im Sinne einer Addressierung modifizierbarer Risikofaktoren ist daher eine frühestmögliche Diagnose der LN einschliesslich Nierenbiopsie und die umgehende Einleitung der Induktionstherapie zu einem Zeitpunkt, zu dem chronische Läsionen nur gering aus- 
geprägt sind, für die Risikominimierung entscheidend. Ferner sollte bei unzureichendem Ansprechen auf die Induktion, insbesondere auch beim Ausbleiben einer Reduktion der Proteinurie auf die o.g. Werte eine Reevaluierung der Therapie möglichst unter Einbeziehung einer erneuten Nierenbiopsie erfolgen und ggf. eine zügige Reinduktion mit einer alternativen Immunsuppression eingeleitet werden, sofern diese auf Basis des Befundes der Rebiopsie Erfolg versprechend erscheint.

\section{Interessenkonflikt}

Peter Oelzner hat Kongressunterstützung durch Pfizer und ein Referentenhonorar von Boehringer erhalten.

\section{Literatur}

[1] Distler O, Assassi S, Cottin V et al. Predictors of progression in systemic sclerosis patients with interstitial lung disease. Eur Respir J 2020; 55. doi.org/10.1183/13993003.02026-2019

[2] Attanasio U, Cuomo A, Pirozzi F et al. Pulmonary hypertension phenotypes in systemic sclerosis: The right diagnosis for the right treatment. Int J Mol Sci 2020; 21. doi:10.3390/ijms21124430

[3] Steele R, Hudson M, Lo E et al. Clinical decision rule to predict the presence of interstitial lung disease in systemic sclerosis. Arthritis Care Res 2012; 64: 519-524

[4] Walker UA, Tyndall A, Czirjak L et al. Clinical risk assessment of organ manifestations in systemic sclerosis: a report from the EULAR Scleroderma Trials and Research group database. Ann Rheum Dis 2007; 66: 754-763

[5] Steen VD, Medsger TA. Changes in causes of death in systemic sclerosis, 1972 - 2002. Ann Rheum Dis 2007; 66: 940-944

[6] Volkmann ER, Tashkin DP, Sim M et al. Short-term progression of interstitial lung disease in systemic sclerosis predicts long-term survival in two independent clinical cohorts. Ann Rheum Dis 2019; 78: 122-130

[7] Fischer A, Patel NM, Volkmann ER. Interstitial lung disease in systemic sclerosis: Focus on early detection and intervention. Open Access Rheumatol 2019; 11: 283-307

[8] Khanna D, Tashkin DP, Denton CP et al. Etiology, risk factors, and biomarkers in systemic sclerosis with interstitial lung disease. Am J Respir Crit Care Med 2020; 201: 650-660

[9] Roofeh A, Jaafar S, Vummidi D et al. Management of systemic sclerosis-associated interstitial lung disease. Curr Opin Rheumatol 2019; 31: 241-249

[10] Goh NS, Hoyles RK, Denton CP et al. Short-term pulmonary function trends are predictive of mortality in interstitial lung disease associated with systemic sclerosis. Arthritis Rheumatol 2017; 69: 1670-1678

[11] Goh NS, Desai SR, Veeraraghavan S et al. Interstitial lung disease in systemic sclerosis. A simple staging system. Am J Respir Crit Care Med 2008; 177: 1248-1254

[12] Distler O, Highland KB, Gahlemann M et al. Nintedanip for systemic sclerosisassociated interstitial lung disease. N Engl J Med 2019; 380: 2518-2528

[13] Almaaitah S, Highland KB, Tonelli AR. Management of pulmonary arterial hypertension in patients with systemic sclerosis. Integr Blood Pres. Control 2020; 13: 15-29

[14] Avouac J, Airo P, Meune C et al. Prevalence of pulmonary hypertension in systemic sclerosis in European Caucasians and metaanalysis of 5 studies. J Rheumatol 2010; 37: 2290-2298
[15] Galie N, Humbert M, Vachiery JL et al. 2015 ESC/ERS guidelines for the diagnosis and treatment of pulmonary hypertension. Eur Heart J 2016; 37: $67-119$

[16] Coghlan JG, Denton CP, Grünig E et al. Evidence-based detection of pulmonary arterial hypertension in systemic sclerosis: The DETECT study. Ann Rheum Dis 2014; 73: 1340-1349

[17] Yaqub A, Chung L. Epidemiology and risk factors for pulmonary hypertension in systemic sclerosis. Curr Rheumatol Rep 2013. doi: $10.1007 / \mathrm{s} 11926-012-0302-2$

[18] Becker MO, Kill A, Kutsche M et al. Vascular receptor autoantibodies in pulmonary arterial hypertension associated with systemic sclerosis. Am J Respir Crit Care Med 2014; 190: 808-817

[19] Sitbon O, Gaine S. Beyond a single pathway: combination therapy in pulmonary arterial hypertension. Eur Respir Rev 2016; 25: 408-417

[20] Nagaraja V. Management of scleroderma renal crisis. Curr Opin Rheumatol 2019; 31: 223-230

[21] Chrabaszcz M, Malysko J, Sikora M et al. Renal involvement in systemic sclerosis. An update. Kidney Blood Press Res 2020; 45: 532-548

[22] Moinzadeh P, Kuhr K, Siegert E et al. Scleroderma renal crisis: Risk factors for an increasingly rare organ complication. J Rheumatol 2020; 47: $241-248$

[23] Bütikofer L, Varisco PA, Distler O et al. ACE inhibitors in SSc patients display a risk factor for scleroderma renal crisis - EUSTAR analysis. Arthritis Res Ther 2020; 22. doi: 10.1186/s13075-020-2141-2

[24] Ranagarajan V, Matiasz R, Freed BH. Cardiac complications of systemic sclerosis and management: recent progress. Curr Opin Rheumatol 2017; 29: 574-584

[25] Komocsi A, Vorobcsuk A, Faludi R et al. The impact of cardiopulmonary manifestations on the mortality of SSc: a systematic review and metaanalysis of observational studies. Rheumatology 2012; 51: 1027-1036

[26] Jaeger VK, Wirz EG, Allanore Y et al. Incidences and risk factors of organ manifestations in the early course of systemic sclerosis: a longitudinal EUSTAR study. PLoS One 2016. doi: 10.1371/journal. pone. 0163894

[27] Bosello S, De Luca G, Berardi G et al. Cardiac troponin T and NT-proBNP as diagnostic and prognostic biomarkers of primary cardiac involvement and disease severity in systemic sclerosis: a prospective study. Eur J Intern Med 2019; 60: 46-53

[28] Morrisroe K, Stevens W, Sahhar J et al. Digital ulcers in systemic sclerosis: their epidemiology, clinical characteristics, and associated clinical and economic burden. Arthritis Res Ther 2019; 21: 299. doi: 10.1186/s13075-019-2080-y

[29] Walker UA, Tyndall A, Czirjak L et al. Clinical risk assessment of organ manifestations in systemic sclerosis: a report from the EULAR scleroderma trials and research group database. Ann Rheum Dis 2007; 66: 754-763

[30] Sunderkötter C, Herrgott I, Brückner C et al. Comparison of patients with and without digital ulcers in systemic sclerosis: detection of possible risk factors. B. J Dermatol 2009; 160: 835-843

[31] Khimdas S, Harding S, Bonner A et al. Associations with digital ulcers in a large cohort of systemic sclerosis: results from the Canadian Scleroderma Research Group Registry. Arthritis Care Res (Hoboken) 2011; 63: 142-149

[32] Silva I, Almeida J, Vasconcelos C. A PRISMA-driven systematic review for predictive risk factors of digital ulcers in systemic sclerosis patients. Autoimm Rev 2015; 14: 140-152

[33] Kowal-Bielecka O, Fransen J, Avouac ] et al. Update of EULAR recommendations for the treatment of systemic sclerosis. Ann Rheum Dis 2017; 76: 1327-1339

[34] Parodis I, Tamirou F, Houssiau FA. Prediction of prognosis and renal outcome in lupus nephritis. Lupus Sci Med 2020. doi: 10.1136/ lupus-2020-000389

[35] Davidson A, Aranow C, Mackay M. Lupus nephritis - challenges and progress. Curr Opin Rheumatol 2019; 31: 682-688 
[36] Danila MI, Pons-Estel G], Zhang J et al. Renal damage is the most important predictor of mortality within the damage index: data from the LUMINA LXIV, a multiethnic US cohort. Rheumatology 2009; 48: 542-545

[37] Derksen RH, Hene RJ, Kater L. The long-term clinical outcome of 56 patients with biopsy-proven lupus nephritis followed at a single center. Lupus 1992; 1: 97-103

[38] Korbet SM, Lewis EJ, Schwartz MM et al. Factors predictive for outcome of severe lupus nephritis. Lupus Nephritis Collaborative Study Group. Am. Kidney Dis 2000; 35: 904-914

[39] Fiehn C, Hajar Y, Mueller K et al. Improved clinical outcome of lupus nephritis during the past decade: importance of early diagnosis and treatment. Ann Rheum Dis 2003; 62: 435-439

[40] Korbet SM, Schwartz MM, Evans ] et al. Severe lupus nephritis: racial differences in presentation and outcome. J Am Soc Nephrol 2007; 18: 244-254

[41] Sun HO, Hu WX, Xie HL et al. Long-term outcome of Chinese patients with membranous lupus nephropathy. Lupus 2008; 17: 56-61

[42] Moon S], Park HS, Kwok SK et al. Predictors of end-stage renal disease and recurrence of lupus activity after initiation of dialysis in patients with lupus nephritis. Clin Exp Rheumatol 2013; 31: 31-39

[43] Haddiya I, Hamzaoui H, Tachfouti N et al. Features and outcomes of lupus nephritis in morocco: analysis of 114 patients. Int J Nephrol Renovasc Dis 2013; 6: 249-258

[44] Yang J, Liang D, Zhang $\mathrm{H}$ et al. Long-term renal outcomes in a cohort of 1814 chinese patients with biopsy-proven lupus nephritis. Lupus 2015; $24: 1468-1478$

[45] Tang Y, Zhang XY, Ji L et al. Clinicopathological and outcome analysis of adult lupus nephritis patients in china: Int Urol Nephrol 2015; 47: 513-520

[46] Urrestarazu A, Otatti G, Silvarino R et al. Lupus nephritis in males: clinical features course, and prognostic factors for end-stage renal disease. Kidney Int Rep 2017; 2: 905-912

[47] Shayakul C, Ong-aj-yooth L, Chirawong P et al. Lupus nephritis in Thailand: clinicopathologic findings and outcome in 569 patients. Am J Kidney Dis 1995; 26: 300-307

[48] Donadio JV Jr, Hart GM, Bergstralh EJ et al. Prognostic determimants in lupus nephritis: a long-term clinicopathologic study. Lupus 1995; 4: 109-115

[49] Shen K, Yu Y, Tang Z et al. The prognosis of biopsy-proven lupus nephritis in chinese patients: long-term follow-up of 86 cases. Chin Med J 1997; 110: 502-507

[50] Mok CC, Wong RW, Lau CS. Lupus nephritis in Southern Chinese patients: clinicopathologic findings and long-term outcome. Am J Kidney Dis 1999; 34: 315-323

[51] Resende AL, Titan SM, Barros RT et al. Worse renal outcome of lupus nephritis in male patients: a case control study. Lupus 2011; 20: 561-567

[52] So NW, Koo BS, Kim YG et al. Predictive value of remission status after 6 month induction therapy in patients with proliferative lupus nephritis: a retrospective analysis. Clin Rheumatol 2011; 30: 1399-1405

[53] Hsu CY, Chiu WC, Yang TS et al. Age- and gender-related long-term outcome in patients with lupus nephritis. Lupus 2011; 20: 1135-1141

[54] Moroni G, Quaglini S, Gravellone L et al. Membranous nephropathy in systemic lupus erythematosus: long-term outcome and prognostic factors of 103 patients. Semin Arthritis Rheum 2012; 41: 642-651

[55] Sui M, Ye X, Ma J et al. Epidemiology and risk factors for chronic kidney disease in chinese patients with biopsy-proven lupus nephritis. Intern Med J 2015; 45: 1167-1172

[56] Malvar A, Pirruccio P, Alberton V et al. Histologic versus clinical remission in proliferative lupus nephritis. Nephrol Dial Transplant 2017; 32: 1338-1344
[57] Yokoyama H, Wada T, Hara A et al. The outcome and a new ISN/RPS classification of lupus nephritis in Japanese. Kidney Int 2004; 66: 2382-2388

[58] Faurschou M, Dreyer L, Kamper AL et al. Long-term mortality and renal outcome in a cohort of 100 patients with lupus nephritis. Arthritis Care Res (Hoboken) 2010; 62: 873-800

[59] Mok CC, Ying KY, Ng WL et al. Long-term outcome of diffuse proliferative lupus glomerulonephritis treated with cyclophosphamide. Am J Med 2006; 355: 25-33

[60] Moroni G, Quaglini S, Galleli B et al. The long-term outcome of 93 patients with proliferative lupus nephritis. Nephrol Dial Transplant 2007; 22: 2531-2539

[61] Dhir V, Aggarwal A, Lawrence A et al. Long-term outcome of lupus nephritis in Asian Indians. Arthritis Care Res (Hoboken) 2012; 64: 713-720

[62] Mahmoud GA, Zayed HS, Ghoniem SA. Renal outcomes among egyptian lupus nephritis patients: a retrospective analysis of 135 cases from a single centre. Lupus 2015; 24: 331-338

[63] Lim CC, Tan HZ, Hao Y et al. Long-term renal outcomes in multi-ethnic Southeast Asians with lupus nephritis: a retrospective cohort study. Internal Medicine Journal 2018; 48: 1117-1123

[64] Ciruelo E, de la Cruz J, Lopez I et al. Cumulative rate of relapse of lupus nephritis after successful treatment with cyclophosphamide. Arthritis Rheum 1996; 39: 2028-2034

[65] Illei CG, Takda K, Parkin D et al. Renal flares are common in patients with severe proliferative lupus nephritis treated with pulse immunosuppressive therapy: long-term followup of a cohort of 145 patients participating in randomized controlled studies. Arthritis Rheum 2002; 46: 995-1002

[66] Chan TM, Tse KC, Tang CS et al. Long-term outcome of patients with diffuse proliferative lupus nephritis treated with prednisolone and oral cyclophosphamide followed by azathioprine. Lupus 2005; 14: 265-272

[67] Cortes-Hernandez J, Ordi-Ros J, Labrador M et al. Predictors of poor renal outcome in patients with lupus nephritis treated with combined pulses of cyclophosphamide and methylprednisolone. Lupus 2003; 12 : 287-296

[68] Fernandes das Neves M, Irlapati RV, Isenberg D. Assessment of long-term remission in lupus nephritis patients: a retrospective analysis over 30 years. Rheumatology 2015; 54: 1403-1407

[69] Laitman RS, Glicklich D, Sablay LB et al. Effect of long-term normalization of serum complement levels on the course of lupus nephritis. Am J Med 1989; 87: 132-138

[70] Barber CE, Geldenhuys L, Hanly JG. Sustained remission in lupus nephritis. Lupus 2006; 15: 94-101

[71] Korbet SM, Lewis E].Collaborative Study Group Severe lupus nephritis: the predictive value of $a \geq 50 \%$ reduction in proteinuria at 6 month. Nephrol Dial Transplant 2013; 28: 2313-2318

[72] Fatemi A, Kazemi M, Sayedbonakdar Z et al. Long-term outcome of biopsy-proven lupus nephritis in Iran. Int J Rheum Dis 2013; 16: 739-746

[73] Vandepapeliere J, Aydin S, Cosyns JP et al. Prognosis of proliferative lupus nephritis subsets in the Louvain Lupus Nephritis inception Cohort. Lupus 2014; 23: 159-165

[74] Dall'Era M, Cisternas MG, Smilek DE et al. Predictors of outcome in lupus nephritis trials: lessons learned from the euro-lupus nephritis cohort. Arthritis Rheumatol 2015; 67: 1305-1313

[75] Tamirou F, D'Cruz D, Sangle S et al. and the MAINTAIN Nephritis Trial Group. Long-term follow-up of the MAINTAIN Nephritis Trial, comparing azathioprine and mycophenolate mofetil as maintenance therapy of lupus nephritis. Ann Rheum Dis 2016; 75: 526-531

[76] Koo HS, Kim S, Chin HJ. Remission of proteinuria indicates good prognosis in patients with diffuse proliferative lupus nephritis. Lupus 2016; 25: 3-11

[77] Fung WA, Su J, Touma Z et al. Predictors of good long-term renal outcomes in lupus nephritis: Results from a single lupus cohort. Biomed Res Int 2017. doi: 10.1155/2017/5312960 